\title{
Intestinal mucosal triacylglycerol accumulation secondary to decreased lipid secretion in obese and high fat fed mice
}

\section{John D. Douglass ${ }^{1}$, Nashmia Malik ${ }^{1}$, Su-Hyoun Chon ${ }^{1+}$, Kevin Wells ${ }^{1}$, Yin Xiu Zhou ${ }^{1}$, Andrew S. Choi ${ }^{1}$, Laurie B. Joseph ${ }^{2}$ and Judith Storch ${ }^{1,3} *$}

\footnotetext{
${ }^{1}$ Department of Nutritional Sciences, School of Environmental and Biological Sciences, Rutgers University, New Brunswick, NJ, USA

2 Department of Pharmacology and Toxicology, Ernest Mario School of Pharmacy, Rutgers University, Piscataway, NJ, USA

${ }^{3}$ Rutgers Center for Lipid Research, School of Environmental and Biological Sciences, Rutgers University, New Brunswick, NJ, USA
}

\section{Edited by:}

Sander Kersten, Wageningen

University, Netherlands

\section{Reviewed by:}

Douglas Mashek, University of

Minnesota, USA

Nicole De Wit, Podiceps BV,

Netherlands

*Correspondence:

Judith Storch, Department of Nutritional Sciences, School of

Environmental and Biological

Sciences, Rutgers University, 96

Lipman Drive, New Brunswick, NJ 08901-8525, USA.

e-mail:storch@aesop.rutgers.edu

\section{${ }^{+}$Present address:}

Su-Hyoun Chon, Johnson and Johnson CPCUS, Skin Research

Center, Skillman, NJ, USA.
The ectopic deposition of fat in liver and muscle during obesity is well established, however surprisingly little is known about the intestine. We used the ob/ob mouse and C57BL6/J mice fed a high fat (HF) diet to examine the effects of obesity and the effects of HF feeding, respectively, on intestinal mucosal triacylglycerol (TG) accumulation. Male C57BL6/J (wildtype, WT) mice were fed low fat (LF; $10 \% \mathrm{kcal}$ as fat) or HF (45\%) diets, and ob/ob mice were fed the LF diet, for 3 weeks. In this time frame, the WT-HF mice did not become obese, enabling independent examination of effects of the HF diet and effects of obesity. Analysis of intestinal lipid extracts from fed and fasted animals demonstrated that the mucosa, like other tissues, accumulates excess lipid. In the fed state, mucosal triacylglycerol (TG) levels were threefold and fivefold higher in the WT-HF and ob/ob mice, respectively, relative to the WT-LF mice. In the fasted state, mucosa from ob/ob mice had threefold higher TG levels relative to WT-LF mucosa. q-PCR analysis of mucosal mRNA from fed state mice showed alterations in the expression of several genes related to both anabolic and catabolic lipid metabolism pathways in WT-HF and ob/ob mice relative to WTLF controls. Fewer changes were found in mucosal samples from the fasted state animals. Remarkably, oral fat tolerance tests showed a striking reduction in the plasma appearance of an oral fat load in the ob/ob and WT-HF mice compared to WT-LF. Overall, the results demonstrate that the intestinal mucosa accumulates excess TG during obesity. Changes in the expression of lipid metabolic and transport genes, as well as reduced secretion of dietary lipid from the mucosal cells into the circulation, may contribute to the TG accumulation in intestinal mucosa during obesity. Moreover, even in the absence of frank obesity, $\mathrm{HF}$ feeding leads to a large decrease in the rate of intestinal lipid secretion.

Keywords: obesity, enterocyte, intestine, lipid, absorption

\section{INTRODUCTION}

The increasing prevalence of metabolic syndrome presents a great challenge to world health. Contributing to the development of coronary heart disease and diabetes mellitus, metabolic syndrome is composed of multiple risk factors that include insulin resistance, dyslipidemia, and central obesity (Eckel et al., 2010). Understanding the connection between dyslipidemia and obesity remains an important goal toward elucidating the pathological mechanisms. In particular, the ectopic deposition of fat seems to play a critical role in tissue dysfunction. Studies show that both obese children and adults exhibit fat deposition in liver and skeletal muscle (Rasouli et al., 2007; Cali and Caprio, 2009; Li et al., 2011). Intrahepatic triacylglycerol (TG) is a known marker for metabolic dysregulation in obese individuals (Fabbrini et al., 2009). Further, intramyocellular TG can result in impaired insulin sensitivity, causing a direct effect on glucose and lipid metabolism (Pan et al., 1997). However, accumulation of lipid in other organs, and its potentially negative effects, has remained relatively unexplored. In particular, little is known about the effects of obesity on the small intestine.
During digestion and absorption dietary fat, composed mostly of TG, is broken down by lipases in the lumen of the digestive tract. The resultant monoacylglycerols and fatty acids (FA) are absorbed by enterocytes in the small intestinal mucosa, where they are reesterified back into TG in the endoplasmic reticulum (ER). The TG is incorporated into chylomicrons (CM), which are trafficked to the Golgi apparatus and secreted into the lymphatic system and eventually the general circulation (Phan and Tso, 2001). There is now a growing body of evidence that newly synthesized TG is not immediately shuttled out of enterocytes, but also stored in lipid droplets (LD). Human studies show that dietary lipids ingested in one meal are present in CM secreted following a second meal (Fielding et al., 1996). Other human studies demonstrate that an oral fat load results in jejunal storage of the ingested lipid that can be released $4 \mathrm{~h}$ later by glucose administration (Robertson et al., 2003). Intraduodenal lipid administration in rats shows that while postprandial TG synthesis is quite rapid, the lipid load can be increased until shuttling of TG from the ER to Golgi stops entirely and a cytoplasmic TG pool is formed that may be an overflow reservoir for later export (Mansbach and Dowell, 2000). 
Finally, in vivo coherent anti-Stokes Raman scattering imaging of murine small intestine shows the existence of cytoplasmic LDs in enterocytes following an oral fat challenge (Zhu et al., 2009). Interestingly, mice fed a high fat (HF) diet had more and larger LDs in enterocytes compared to low fat diet controls (Lee et al., 2009; Zhu et al., 2009).

We used two murine models to explore the effects of obesity and diet on the small intestine: $o b / o b$ mice and HF diet fed C57BL6/J mice. Owing to a single gene mutation that prevents the production of the adipokine leptin, the $o b / o b$ mouse is a model for type 2 diabetes that exhibits hyperglycemia and marked obesity (Zhang et al., 1994; Drel et al., 2006). This is largely due to dysregulation of central appetite signaling which results in hyperphagia, as well as dysfunctions in FA and TG metabolism in peripheral tissues (Friedman and Halaas, 1998; Unger et al., 1999). We also used C57BL6/J mice to examine the effects of HF feeding on mucosal TG. We hypothesized that in obese animals the intestinal mucosa, like liver, and skeletal muscle, would accumulate lipids. In the present study, we report that obese mice accumulate mucosal TG in the fed and fasted state. This TG deposition is accompanied by changes in the mucosal expression of several lipogenic and lipid trafficking genes. Furthermore, both the ob/ob and the WTHF mice display a marked reduction in TG secretion following an oral lipid load, relative to WT-LF mice. These results expand our understanding of obesity and reinforce the idea that tissue fat accumulation is often concurrent with metabolic dysfunction.

\section{MATERIALS AND METHODS \\ ANIMALS, DIET, SURGICAL PROCEDURES, AND TISSUE COLLECTION}

Thirty C57BL/6J wild-type (WT) male mice and 13 ob/ob male mice were obtained from Jackson Laboratories (Bar Harbor, ME, USA). All mice were 8 weeks old upon arrival and weighed $22-27 \mathrm{~g}$ (WT) or 47-54 g (ob/ob). The mice were housed three per cage and fed Purina 5015 rodent chow (60\% carbohydrate, 12\% fat, 28\% protein by kcal) for a week before the start of the study. For the 3-week feeding period, $15 \mathrm{WT}$ mice and $13 \mathrm{ob} / \mathrm{ob}$ mice were fed a $10 \%$ kcal low fat semipurified diet (D12325, Research Diets, Inc., New Brunswick, NJ, USA; Table 1). The remaining 15 WT mice were fed a $45 \% \mathrm{kcal} H F$ semipurified diet (D12327, Research Diets, Inc., New Brunswick, NJ, USA; Table 1). The WT-LF mice served as controls for dietary fat content (versus WT-HF fed mice), and for genotype (versus $o b / o b$-LF-fed mice). These three groups were used for all analyses.

At the end of the 3-week feeding period, body composition was analyzed by magnetic resonance imaging using an EchoMRI-100 (Echo Medical Systems, Houston, TX, USA). Food was removed from the mice cages $12 \mathrm{~h}$ prior to sacrifice (fasted state) or the mice were given ad libitum food access (fed state). Unless otherwise noted, tissue collections were performed between 9 and 11 am. Before sacrifice, the mice were injected intraperitoneally with a ketamine-xylazine-ace promazine cocktail $(54.5 / 5.45 / 0.8 \mathrm{mg} / \mathrm{kg}$, respectively) to induce deep anesthesia, followed by exsanguination. The entire small intestine was excised, rinsed twice with saline $(0.85 \% \mathrm{NaCl})$, and the mucosa harvested by scraping with a glass slide. The mucosa samples were immediately weighed in polypropylene tubes and frozen in dry-ice ethanol. The liver was excised, weighed, and frozen in dry-ice ethanol. The intestinal
Table 1 | Diet composition.

\begin{tabular}{|c|c|c|c|c|}
\hline & \multicolumn{2}{|c|}{ D12325 } & \multicolumn{2}{|c|}{ D12327 } \\
\hline & g \% & kcal \% & g \% & kcal \% \\
\hline \multicolumn{5}{|l|}{ DIET } \\
\hline Protein & 19 & 20 & 23 & 20 \\
\hline Carbohydrate & 68 & 70 & 46.1 & 40 \\
\hline Fat & 4 & 10 & 20.4 & 39.9 \\
\hline Total & & 100 & & 100 \\
\hline $\mathrm{kcal} / \mathrm{gm}$ & 3.86 & & 4.6 & \\
\hline \multicolumn{5}{|l|}{ INGREDIENT } \\
\hline Casein & 200 & 800 & 200 & 800 \\
\hline DL-methionine & 3 & 12 & 3 & 12 \\
\hline Sucrose & 700 & 2800 & 396 & 1584 \\
\hline Cellulose & 50 & 0 & 50 & 0 \\
\hline Soybean oil & 45 & 405 & 45 & 405 \\
\hline Coconut oil & & & 135 & 1215 \\
\hline Mineral Mix & 35 & 0 & 35 & 0 \\
\hline Calcium carbonate & 5 & 0 & 5 & 0 \\
\hline Vitamin mix & 10 & 40 & 10 & 40 \\
\hline Choline bitartrate & 2 & 0 & 2 & 0 \\
\hline FD\&C blue dye \#1 & 0.05 & 0 & 0.05 & 0 \\
\hline FD\&C red dye \#40 & & & 0.05 & 0 \\
\hline Total & 1050 & 4057 & 881.1 & 4056 \\
\hline
\end{tabular}

mucosa samples were immediately homogenized followed by subsequent lipid and RNA extractions on the same day of the experiment. The liver samples were stored at $-80^{\circ} \mathrm{C}$ until later analysis. All animal procedures were approved by the Rutgers University Animal Use Protocol Review Committee and conformed to the National Institutes of Health Guide for the Care and Use of Laboratory Animals.

\section{LIPID EXTRACTION AND TG ANALYSIS}

Liver and mucosa samples were homogenized with $1 \times \mathrm{PBS}$ pH 7.4 in $20 \times$ and $10 \times \mathrm{ml} / \mathrm{g}$ of sample, respectively, on ice with a Dounce homogenizer and a Wheaton overhead stirrer at 5,000 rpm. Total tissue protein concentration was determined by Bradford assay (Bradford, 1976). The homogenate was diluted to $1 \mathrm{mg}$ protein $/ \mathrm{ml}$ in PBS and lipid extractions were performed on $1 \mathrm{ml}$ of diluted sample by the Folch procedure (Folch et al., 1957). Lipid extracts and triolein standards were spotted on Silica Gel G TLC plates and separated by a non-polar solvent system (hexane-diethyl etheracetic acid, 70:30:1; v/v). Lipids were visualized by iodine vapor and quantitation of TG determined using ImageJ (Bethesda, MD, USA), as previously described (Storch et al., 2008).

\section{HISTOLOGICAL EXAMINATION OF MUCOSAL LIPID}

Mice were maintained and fed as described in the previous section. All mice were given ad libitum access to food and water prior to necropsies (fed state), which were performed between 9 and 11 am. The mice were first anesthetized as described above, followed by exsanguination. The small intestine was removed, rinsed with buffered PBS, trimmed, tied off, and filled with $4 \%$ paraformaldehyde in PBS for $2 \mathrm{~h}$ at room temperature. The lumen was then 
rinsed with $2 \%$ sucrose in $\mathrm{PBS}$, cut into $7 \mathrm{~mm}$ pieces and embedded in $10 \mathrm{~mm}$ Tissue-Tek Cryomolds (Sakura Finetek, Torrance, CA, USA) filled with Tissue-Tek O.C.T. Compound (Sakura Finetek, Torrance, CA, USA). Molds containing OCT and tissue were snap frozen in liquid nitrogen and stored at $-80^{\circ} \mathrm{C}$ until use.

For Oil Red $\mathrm{O}$ analysis, $10 \mu \mathrm{m}$ frozen sections were cut on a Microm HM505E cryostat using Edge-Rite Low Profile Microtome Blades (Richard-Allan Scientific, Kalamazoo, MI, USA) and mounted to Superfrost Plus Micro Slides (VWR, Radnor, PA, USA). Sectioned tissue was stored at $-20^{\circ} \mathrm{C}$. Tissue was removed from the $-20^{\circ} \mathrm{C}$ and brought to room temperature for $15 \mathrm{~min}$ prior to Oil Red O staining. Oil Red O (Fisher Scientific, Pittsburgh, NJ, USA) was prepared according to Humason (1972). Immediately prior to use the stock solution was diluted with distilled deionized water $(6: 4, \mathrm{~V} / \mathrm{V})$ and filtered through a $0.2-\mu \mathrm{m}$ filter. Tissue sections were post-fixed with 10\% formalin (Fisher Scientific, Pittsburgh, PA, USA) for $15 \mathrm{~min}$, dipped in 60\% isopropanol for $30 \mathrm{~s}$, and stained with Oil Red $\mathrm{O}$ for $30 \mathrm{~min}$. Tissue was destined with $60 \%$ isopropanol, rinsed under running tap for $2 \mathrm{~min}$, and counterstained with hematoxylin (Invitrogen, Camarillo, CA, USA). Oil Red O sections were preserved with glycerin (Fisher Scientific, Pittsburgh, NJ, USA) under nail polish sealed cover glass (Fisher Scientific, Pittsburgh, PA, USA). Random sections were analyzed and photographed using an Olympus BX51 microscope equipped with a DP71 digital camera.

\section{RNA EXTRACTION AND QUANTITATIVE RT-PCR ANALYSIS}

Total mRNA was extracted from intestinal mucosa using a modified method from Chomczynski and Sacchi (Chon et al., 2007) and analyzed as previously described (Lagakos et al., 2011). In brief, the tissues were homogenized in $4 \mathrm{M}$ guanidinium thiocyanate with an Ultra-Turrax IKA-Werke (Wilmington, DE, USA). Total RNA was isolated by phenol extraction, followed by precipitation and washing with ethanol. The RNA was further purified by removal of genomic DNA by DNase digest and RNeasy cleanup kit (Qiagen, Valencia, CA, USA). The integrity of the RNA was assessed by gel electrophoresis and visualization of the $18 \mathrm{~S}$ and $23 \mathrm{~S}$ rRNA subunits. Reverse transcription was performed on $2 \mu \mathrm{g}$ of RNA using a high capacity cDNA kit (Promega, Madison, WI, USA). Primer sequences were determined using the NCBI gene database and ordered from Sigma Aldrich (St. Louis, MO, USA) for the following primers shown in Table 2: $\beta$-actin, monoacylglycerol acyltransferase 2 (MGAT2), diacylglycerol acyltransferase 1 (DGAT1), diacylglycerol acyltransferase 2 (DGAT2), monoacylglycerol lipase (MGL), glycerophosphate acyltransferase 3 (erGPAT3), mitochondrial glycerophosphate acyltransferase (mtGPAT), CD36, microsomal triacylglycerol transfer protein (MTP), liver fatty acid binding protein (LFABP), intestinal fatty acid binding protein (IFABP), carnitine palmitoyltransferase 1 (CPT1), acyl-CoA oxidase (ACO), fatty acid synthase (FASN), and acetyl-CoA carboxylase 1 (ACC1). The efficiency $(100 \pm 5 \%)$ of each PCR primer set was first assessed by standard curve. Real time PCR (RT-PCR) was performed in triplicate using the ddCT method on the ABI 7300 PCR instrument (Applied Biosystems, Foster City, CA, USA). Each reaction contained $80 \mathrm{ng}$ of $\mathrm{cDNA}, 250 \mathrm{nM}$ of each forward and reverse primer, and $12.5 \mu$ l of POWER SYBR Green Master Mix (Applied Biosystems, Foster City, CA, USA) in a total volume of $25 \mu \mathrm{l}$.
Table 2 | Primer sequences used for qRT-PCR analyses.

\begin{tabular}{|c|c|}
\hline Gene & Primer sequence (forward and reverse) \\
\hline \multirow[t]{2}{*}{$\beta$-actin } & 5'-GGCTGTATTCCCCTCCATCG-3' \\
\hline & 5'-CCAGTTGGTAACAATGCCATGT-3' \\
\hline \multirow[t]{2}{*}{ MGAT2 } & 5'-CGGAGGTGGACAACCTAACG-3' \\
\hline & 5'-TGAGGTATTCCGGCCTGTTAT-3' \\
\hline \multirow[t]{2}{*}{ DGAT1 } & 5'-TGTTCAGCTCAGACAGTGGTT-3' \\
\hline & 5'-CCACCAGGATGCCATACTTGAT-3' \\
\hline \multirow[t]{2}{*}{ DGAT2 } & 5'-TTCCTGGCATAAGGCCCTATT-3' \\
\hline & 5'-AGTCTATGGTGTCTCGGTTGAC-3' \\
\hline \multirow[t]{2}{*}{ MGL } & 5'-CAGAGAGGCCCACCTACTTTT-3' \\
\hline & 5'-ATGCGCCCCAAGGTCATATTT-3' \\
\hline \multirow[t]{2}{*}{ erGPAT3 } & 5'-TATCCAAAGAGATGAGTCACCCA-3' \\
\hline & 5'-CACAATGGCTTCCAACCCCTT-3' \\
\hline \multirow[t]{2}{*}{ mtGPAT } & 5'-CTGCTTGCCTACCTGAAGACC-3' \\
\hline & 5'-GATACGGCGGTATAGGTGCTT-3' \\
\hline \multirow[t]{2}{*}{ CD36 } & 5'-TCCCССTACTAGAAGAAGTGGG-3' \\
\hline & 5'-TCCAACAGATTGGTTTCGTTCA-3' \\
\hline \multirow[t]{2}{*}{ MTP } & 5'-CTCTTGGCAGTGCTTTTTCTCT-3' \\
\hline & 5'-GAGCTTGTATAGCCGCTCATT-3' \\
\hline \multirow[t]{2}{*}{ LFABP } & 5'-GGGGGTGTCAGAAATCGTG-3' \\
\hline & 5'-CAGCTTGACGACTGCCTTG-3' \\
\hline \multirow[t]{2}{*}{ IFABP } & $5^{\prime}$-GTGGAAAGTAGACCGGAACGA-3' \\
\hline & 5'-CCATCCTGTGTGATTGTCAGTT-3' \\
\hline \multirow[t]{2}{*}{ CPT1 } & 5'-AGCACACCAGGCAGTAGCTT-3' \\
\hline & 5'-AGGATGCCATTCTTGATTCG-3' \\
\hline \multirow[t]{2}{*}{$\mathrm{ACO}$} & 5'-ATATTTACGTCACGTTTACCCCGG-3' \\
\hline & 5'-GGCAGGTCATTCAAGTACGACAC-3' \\
\hline \multirow[t]{2}{*}{ FASN } & 5'-AGGTGGTGATAGCCGGTATGT-3' \\
\hline & 5'-TGGGTAATCCATAGAGCCCAG-3' \\
\hline \multirow[t]{2}{*}{$\mathrm{ACC} 1$} & 5'-ATGGGCGGAATGGTCTCTTTC-3' \\
\hline & 5'-TGGGGACCTTGTCTTCATCAT-3' \\
\hline
\end{tabular}

$\beta$-actin was used as the endogenous control for each standard and the relative quantitation of each gene was determined with respect to the average of the WT-LF mice.

\section{ORAL FAT TOLERANCE TEST}

Following the 3-week feeding study the mice were fasted for $16 \mathrm{~h}$ overnight. All OFTT were conducted from 9 am to $1 \mathrm{pm}$. The mice were weighed and at $t_{0}$ were injected with $500 \mathrm{mg} / \mathrm{kg}$ body weight of Tyloxapol (Triton WR-1339) to block peripheral lipoprotein clearance. Thirty minutes following Tyloxapol injection, $300 \mu \mathrm{l}$ of olive oil (OO) was given by orogastric gavage. Blood samples were collected from the tail at time $=0\left(t_{0}\right), 30,60,90,150,240 \mathrm{~min}$. The amount of TG present in blood plasma at each time point was determined using a Wako L-Type TG-H kit (Richmond, VA, USA) and performed in duplicate in a microplate reader.

\section{STATISTICAL METHODS}

Unless otherwise noted, all group data are shown as average \pm SEM. Statistical comparisons were determined using a twosided Student's $t$-test or one-way ANOVA with Tukey's post hoc comparison. Differences were considered significant at $p<0.05$. 


\section{RESULTS}

\section{HIGH FAT FED AND OB/OB MOUSE MODELS}

As expected at baseline the WT mice had a substantially lower body weight than their age-matched $o b / o b$ counterparts (Figure 1A). Following the 3-week feeding period, the $o b / \mathrm{ob}$ maintained their weight. The WT-LF and WT-HF groups gained approximately $3 \mathrm{~g} /$ mouse (Figure 1A). At the end of the 3-weeks, body composition was assessed by MRI and showed that the $o b / o b$ mice had a significantly higher percentage of total body fat than both the WT-LF and WT-HF groups (Figure 1B). There was no significant difference in total body fat percentage between the WT-LF and WT-HF groups. The ob/ob group also displayed significantly more epididymal fat as a percentage of their body weight than either WT group (Figure 1C). While there was a trend of higher percent epididymal fat in the WT-HF compared to WT-LF mice, it did not reach statistical significance. The hedonic mouse strain C57BL6/J tends to develop obesity and hyperglycemia (Petro et al., 2004). That the WT-HF mice did not gain weight relative to the WTLF group is in contrast to our previous studies using older mice (Chon et al., 2007), however, this allowed a comparison between the effects of HF feeding in the absence of obesity, to the effects of genetically mediated obesity.

\section{TG CONTENT OF INTESTINAL MUCOSA AND LIVER}

Hepatic TG levels were $120 \pm 29,129 \pm 35$, and $3698 \pm 323 \mu \mathrm{g} / \mathrm{mg}$ protein for the WT-LF, WT-HF, and $o b / o b$ mice, respectively, in the fed state. We also quantified the amount of TG in the intestinal mucosa of fed and fasted mice (Figure 2). In fasted mice, the ob/ob group had approximately threefold higher mucosal TG compared the WT-LF and WT-HF groups $(p<0.01)$. Thus, in the ob/ob genetic obesity model, intestinal mucosa has increased TG levels even in the fasted state. In the fed state, mucosal TG levels in ob/ob were fivefold greater than in the WT-LF mice $(p<0.01)$. In WT animals, a threefold increase in mucosal TG was found in the fed state in HF animals, however in the fasted state there were no differences in mucosal TG levels between HF- and LF-fed mice. Fecal fat levels were $<4 \%$ by weight in all groups (not shown).

Neutral lipid accumulation in duodenal sections from the proximal small intestine of fed mice was visualized using ORO staining.
In keeping with the biochemical analysis, pronounced staining was observed in $o b / o b$ mucosa, and greater staining was found in WT-HF compared to WT-LF mucosa (Figures 3A-C). We noticed during sectioning and histological preparation that the $o b / o b$ mucosal samples were more fragile than either of the WT samples, as seen by decreased mucosal integrity (Figure 3C).

\section{LIPID METABOLIC GENE EXPRESSION IS ALTERED IN OBESE INTESTINAL MUCOSA}

To further understand the changes that may account for increased enterocyte TG storage, we quantified the relative expression of 14 lipid metabolic and transport genes in the intestinal mucosa of fed and fasted mice using RT-PCR. In fasted WT-HF mice DGAT2, MGL, MTP, and FASN expression were significantly greater than in fasted WT-LF controls (Figure 4A). In the fed state these same genes as well as erGPAT3, CD36, CPT1, ACO, and ACC1 were significantly increased in the WT-HF group, while MGAT2 transcript levels were significantly decreased. In both the fasted and fed states, mtGPAT, DGAT1, IFABP, and LFABP were not significantly different between the WT mice fed either a low fat or HF diet.

In contrast to the WT-HF group, in fasted $o b / o b$ mice, many of the genes analyzed were significantly down-regulated relative to the WT-LF controls (Figure 4B). However in the fed state, similar to what was observed in the WT-HF group, most of the genes analyzed were increased relative to WT-LF mice, including a robust upregulation of CPT1. These results indicate that both the HF diet and genotype influence the expression of genes in intestinal mucosa involved in TG synthesis, chylomicron secretion, and the uptake, oxidation, and de novo synthesis of FAs. It is therefore possible that gene expression differences in obese mice may, in part, contribute to the observed TG accumulation in $o b / o b$ and WT-HF mouse intestine (Figures $\mathbf{2}$ and $\mathbf{3}$ ).

\section{DECREASED INTESTINAL TG SECRETION IN OB/OB AND WT-HF MICE}

We challenged the three groups with an OFTT to determine their capacity to absorb a bolus of $\mathrm{OO}$ and release it into the bloodstream. Both the WT-HF and $o b / o b$ mice had dramatic reductions in appearance of the oil load as serum TG over the 4 -h test. The
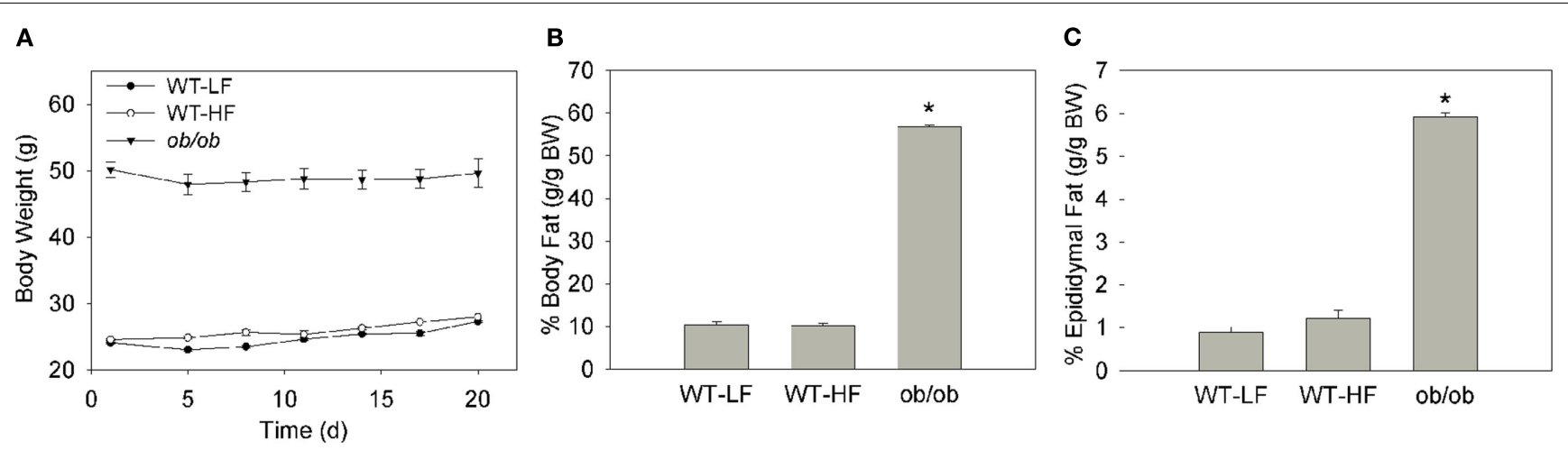

FIGURE 1 | Weight and body fat of mouse models. (A) Average body weight of mice over the 3-week feeding study. Error bars may not be visible within data point. (B) Percent body fat as determined by EchoMRI. (C)
Percent of total body weight as epididymal fat determined by tissue weight. Data represent average \pm SEM, $p<0.05$ or lower versus WT-LF, $n=5-6$ for WT, $n=3$ for $o b / o b$. 
differences between groups increased over time up to $t_{240}$, at which point serum TG levels for WT-LF, WT-HF, and $o b / o b$ mice were $1180 \pm 2,359 \pm 133$, and $134 \pm 17 \mathrm{mg} / \mathrm{dl}$, respectively (Figure 5). The markedly slower rates of TG secretion in the WT-HF mice and the $o b / o b$ mice suggest that obesity and HF feeding are accompanied by intestinal dysfunction. The reduced rate of intestinal lipid secretion likely contributes to increased storage of dietary fat in enterocyte LDs, resulting in increased mucosal TG accumulation (Figures 2 and 3).

\section{DISCUSSION}

In obesity, excess fat deposition occurs not only in adipocytes but also in liver and skeletal muscle. In the present study, we explored the effects of obesity and HF feeding on the small intestine, and found that mucosal TG accumulation is accompanied by changes in lipid metabolic gene expression and markedly diminished secretion of dietary lipids.

As expected the $o b / o b$ mice displayed significantly higher liver TG content. Ectopic fat deposition in the liver, or hepatosteatosis, is commonly associated with obesity and can progress into fibrosis and severely compromised liver function, eventually leading to cirrhosis and hepatocarcinoma (Festi et al., 2004; Janardan and Rao, 2006). The delayed lipoprotein secretion observed in the



FIGURE 2 |TG content in liver and intestinal mucosa. Intestinal mucosa TG content in fed and $12-\mathrm{h}$ fasted mice. Average \pm SEM, ${ }^{*} p<0.05$ or lower versus fed or fasted WT-LF, $n=4-6$ for WT, $n=3-4$ for $o b / o b$. present study suggests a similar dysfunctional relationship to enterocyte lipid accumulation and tissue function. Inflammation is also likely to play a contributory role in the intestinal dysfunction, as it has been recently shown that obese rats fed a HF diet exhibit gut inflammation coupled with alterations in tight junctions and increased intestinal permeability (de La Serre et al., 2010).

In the fed state, the $o b / o b$ mucosa contained fivefold higher TG levels than WT mucosa. Notably, we found threefold higher TG levels in fasted ob/ob mice as well. A recent study by Zhu et al. (2009). showed that in lean WT mice the enterocyte LDs present after an OO gavage of are almost entirely depleted $12 \mathrm{~h}$ later. This is in agreement with the absence of TG accumulation in the 12$h$ fasted WT-LF group. The results indicate that mucosal LDs in obese mice persist even after a long period of time following lipid ingestion. However, it remains to be determined if this is a result of obesity per se, or whether the absence of leptin has a specific effect in the small intestine to promote an energy sparing phenotype. Interestingly, Iqbal et al. (2010). reported that leptin receptor Bdeficient mice also had elevated intestinal mucosal TG levels. Other hormones may also play a role, particularly insulin, which has been reported to produce effects in the small intestine. For example, it has been reported that adolescents with diabetes mellitus exhibit lipid accumulation within proximal jejunum mucosa (Bobo et al., 1977). Insulin has also been shown to decrease chylomicron production in human jejunal explants (Loirdighi et al., 1992), and rodent studies show many effects of insulin resistance on insulin signaling in enterocytes, including increased de novo lipogenesis and altered GLUT2 translocation (Haidari et al., 2002; Tobin et al., 2008). Thus, the mucosal lipid accumulation shown here in $o b / o b$ mice may, in part, be secondary to insulin resistance in this mouse model.

Increased postprandial TG biosynthesis and storage, as well as reduced $\mathrm{CM}$ secretion, are both potential contributors to the increased intestinal lipid content observed herein. TG biosynthesis in enterocytes can occur via MGAT2 and DGAT1/2 activity or the glycerol-3-phosphate pathway, with the former pathway thought to contribute more than $75 \%$ of TG resynthesis (Johnston et al., 1967). We found increased mRNA abundance of most enzymes involved in both pathways in the fed $o b / o b$ mice. Overexpression of DGAT2, but not DGAT1, in mouse liver promotes LD formation, suggesting a dominant role for DGAT2 in TG storage (Yamazaki et al., 2005). DGAT1 ${ }^{-1-}$ mice display reduced CM secretion, supporting a preferential export of DGAT1-catalyzed TG. In the fed $o b / o b$ mice, the DGAT2 expression increase was modestly


FIGURE 3 | Lipid accumulation in fed state proximal intestinal mucosa. (A) LF-fed WT mice (B) HF fed WT mice (C) LF-fed ob/ob mice. 40× magnification, $10 \mu \mathrm{m}$ section, ORO, and H\&E stain, as described in Section "Materials and Methods." 



FIGURE 4 | Relative quantitation of mRNA expression of lipid metabolic and transport genes. (A) Fasted and fed WT-HF mice relative to fasted and fed WT-LF mice. (B) Fasted and fed $o b / o b$ mice relative to fasted and fed WT-LF mice. Average \pm SEM, ${ }^{*} p<0.05$ or lower versus fasted WT-LF, ${ }^{*} p<0.05$ or lower versus fed WT-LF, $n=3-6$ per group.

greater than the increase in DGAT1 expression $(1.96 \pm 0.14$ and $1.54 \pm 0.07$, respectively, $p=0.04$ ), although both were significantly increased relative to fed WT-LF mice. Pharmacological inhibition of intestinal lipoprotein secretion in rats results in markedly increased intestinal lipid content, indicating a primary role for CM biogenesis and secretion in regulating intracellular lipid levels (Tso et al., 1980). MTP is important as an intracellular chaperone in lipoprotein biogenesis, and its function and activity may be altered in the obese intestine (Atzel and Wetterau, 1993; Swift et al., 2005). In the present study we found that MTP expression was unchanged in the $o b / o b$ mice in the fasted state, despite the presence of mucosal TG; expression in fed $o b / o b$ mice was increased 1.3-fold, a change that appears to be insufficient for efficient export of a lipid load.

It is interesting that many of the mucosal genes analyzed were lower in fasted $o b / o b$ mice compared to fasted WT-LF mice. While this would appear to oppose the obese phenotype of these mice, it is worth noting that that expression of adipogenic enzymes in

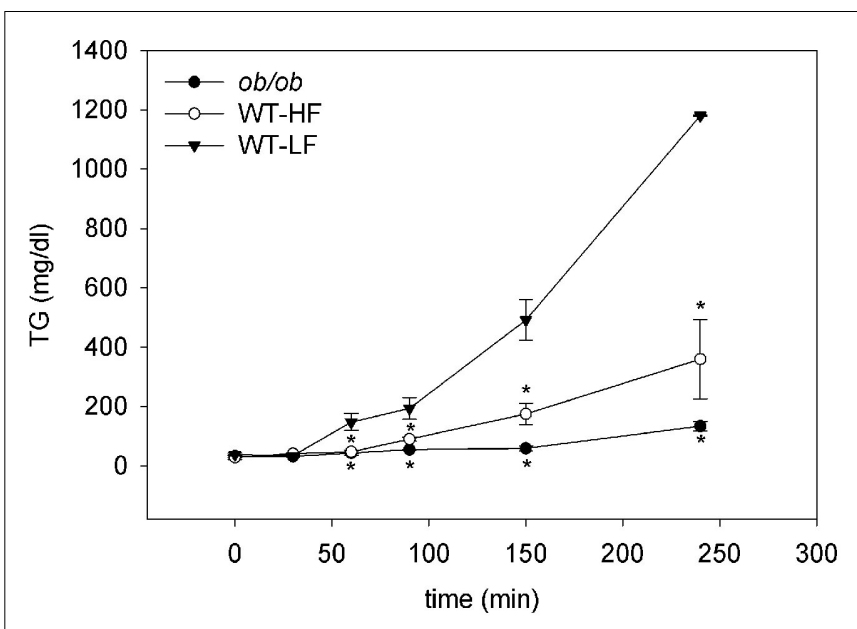

FIGURE 5 | Oral fat tolerance tests. Fasted mice given the LPL-inhibitor Tyloxapol were gavaged with an olive oil bolus as described in Section "Materials and Methods." Average \pm SEM, $n=4$ per group, ${ }^{*} p<0.05$ or lower versus WT-LF.

adipose tissues of $o b / o b$ mice is also reported to be significantly decreased, suggesting that absence of functional leptin signaling induces an energy sparing state in peripheral tissues (Nadler et al., 2000).

De novo FA synthesis could also be contributing to the observed mucosal TG accumulation. Although the contribution of endogenous synthesis to total intestinal TG is thought to be small, it has been shown that FA synthesis is increased twofold in the small intestine of $o b / o b$ mice relative to lean WT mice (Memon et al., 1994). We found increased ACC1 expression in fed ob/ob mice, and a trend toward increased FASN. It should be noted that our $o b / o b$ mice consumed a high sucrose LFD, thereby providing an abundant supply of substrate for lipogenesis.

Markedly diminished secretion of a dietary lipid bolus was found not only in the ob/ob but also in the WT-HF group. Despite having a similar weight and body composition to the WTLF mice, the WT-HF mice had a much lower rate of secretion, implying that enterocyte dysfunction may precede the accumulation of mucosal lipids. The HF diet may also be an independent contributor to the secretion defect. In human studies, HF diet interventions for as little as 3 days as well as for 2 weeks, have been shown to significantly decrease gastrointestinal transit time (Cunningham et al., 1991; Castiglione et al., 2002; Clegg et al., 2011). Additionally, rodent studies show that increased gastric emptying as a result of chronic HF feeding may be attributed, in part, to impaired cholecystokinin (CCK) signaling (Covasa and Ritter, 2000). Thus, it is possible that the HF feeding in the present WT-HF group leads to an acute lipid load in the proximal small intestine secondary to increased gastric emptying. In turn, this may underlie the increased postprandial lipid accumulation and upregulation of mucosal genes such as DGAT2 and CD36 in the intestinal mucosa of fed WT-HF mice. Adaptation to the HF diet by persistently increased gastric emptying rates may therefore contribute to the secretion dysfunction observed in the OFTT. 
The reduced rate of intestinal TG secretion may also be secondary, at least in part, to changes in mucosal lipid metabolism. The increased expression of erGPAT3 coupled with the reduced expression of MGAT2 suggests the potential switch in anabolic metabolism from TG production to phospholipid biosynthesis, which may contribute to reduced TG available for secretion. In addition, the increased CPT1 and ACO mRNA in fed $o b / o b$ and WT-HF samples, relative to WT-LF, suggest an increased level of lipid oxidation. Although the levels of mucosal FA oxidation are quite low (Windmueller and Spaeth, 1978; Lagakos et al., 2011), it is possible that an increase in FA oxidation could play a small role in reducing the secreted TG pool.

It is important to note that very little is known about the regulation of mucosal lipid metabolic gene expression. We showed previously that, unlike in the liver where transcriptional regulation of expression of MGAT and MGL were found, in the intestinal mucosa both of these genes appeared to be highly regulated by post-transcriptional mechanisms (Chon et al., 2007). Thus, in future studies we will determine the effects of obesity and HF feeding on the protein expression and activities of lipid metabolic and transport proteins, to gain further insight into the mechanisms by which the mucosa accumulates increased TG and develops reduced postprandial TG secretion.

The dramatically lower rates of lipid secretion in $o b / o b$ and HF fed mice are surprising in light of multiple studies that show increased postprandial lipemia in obese subjects (Lewis et al., 1990; Mekki et al., 1999). An important factor may be the contribution of the liver to plasma TG, as it has been shown that hepatic lipoprotein production accounts for a significant part of postprandial TG levels (Lewis et al., 1990). While we cannot rule out the influence of endogenous lipoprotein production by the liver, the OFTT protocol uses an intentionally large bolus of $\mathrm{OO}$ to focus on intestinal lipid secretion and minimize the hepatic contribution. In keeping

\section{REFERENCES}

Atzel, A., and Wetterau, J. R. (1993). Mechanism of microsomal triglyceride transfer protein catalyzed lipid transport. Biochemistry 32, 10444-10450.

Bobo, R. C., Partin, J. C., Schubert, W. K., and Saalfeld, K. (1977). Abnormal lipid accumulation within the small intestinal mucosa of children with juvenile-onset diabetes mellitus. Am. J. Dis. Child. 131, 962-969.

Bradford, M. M. (1976). A rapid and sensitive method for the quantitation of microgram quantities of protein utilizing the principle of protein-dye binding. Anal. Biochem. $72,248-254$.

Cali, A. M., and Caprio, S. (2009). Ectopic fat deposition and the metabolic syndrome in obese children and adolescents. Horm. Res. 71(Suppl. 1), 2-7.

Castiglione, K. E., Read, N. W., and French, S. J. (2002). Adaptation to high-fat diet accelerates emptying of fat but not carbohydrate test meals in humans. Am. J. Physiol. Regul. Integr. Comp. Physiol. 282, R366-R371.

Chon, S. H., Zhou, Y. X., Dixon, J., and Storch, J. (2007). Intestinal monoacylglycerol metabolism: developmental and nutritional regulation of monoacylglycerol lipase and monoacylglycerol acyltransferase. J. Biol. Chem. 282, 33346-33357.

Clegg, M. E., McKenna, P., McClean, C., Davison, G. W., Trinick, T., Duly, E., and Shafat, A. (2011). Gastrointestinal transit, post-prandial lipaemia and satiety following 3 days high-fat diet in men. Eur. J. Clin. Nutr. 65, 240-246.

Covasa, M., and Ritter, R. C. (2000). Adaptation to high-fat diet reduces inhibition of gastric emptying by CCK and intestinal oleate. Am. J. Physiol. Regul. Integr. Comp. Physiol. 278, R166-R170.

Cunningham, K. M., Daly, J., Horowitz, M., and Read, N. W. (1991). Gastrointestinal adaptation to diets of differing fat composition in human volunteers. Gut 32, 483-486.

with the present results, Ji and Friedman (2008) found a significant inverse correlation between body weight increase and changes in plasma TG levels following an intragastric corn oil gavage in rats.

It is notable that diminished lipid secretion is also observed by Uchida et al. (2012) in the current issue, in both diet-induced obese (DIO) and $o b / o b$ mice. They found that plasma TG appearance following an acute intragastric fat challenge was reduced in the $o b / o b$ and DIO mice relative to lean LFD-fed controls. This was accompanied by postprandial hypertriglyceridemia and a delayed TG peak in oral fat challenges without Tyloxapol, indicating a role for hepatic lipid secretion and lipoprotein clearance in determining circulating TG levels. Differences between our studies and theirs include the diet composition and length of feeding. Nevertheless, the intestinal lipid secretion results concur, demonstrating that reduced TG secretion is a persistent defect that can be observed after both 3 weeks of HF feeding, in the absence of obesity, and after 6 weeks of HF feeding, when DIO is present, as well as under varying dietary fat composition, suggesting that the amount rather than the type of fat in the diet is a primary determinant of intestinal lipid secretion rate.

In summary, we report for the first time that the intestinal mucosa accumulates excess TG during obesity. Both alterations in the expression of lipid metabolic and transport genes, as well as reduced secretion of dietary lipid, may contribute to the TG accumulation in intestinal mucosa during HF feeding and obesity.

\section{ACKNOWLEDGMENTS}

These studies were supported by National Institutes of Health grant DK38389 (Judith Storch) and by funds from the New Jersey Agricultural Experiment Station. The authors thank Ms. Jessica Cervelli and Ms. Gabriella Composto for expert assistance with tissue histology.

de La Serre, C. B., Ellis, C. L., Lee, J., Hartman, A. L., Rutledge, J. C., and Raybould, H. E. (2010). Propensity to high-fat diet-induced obesity in rats is associated with changes in the gut microbiota and gut inflammation. Am. J. Physiol. Gastrointest. Liver Physiol. 299, G440-G448.

Drel, V. R., Mashtalir, N., Ilnytska, O., Shin, J., Li, F., Valeriy, V., Lyzogubov, V., and Obrosova, I. G. (2006). The leptin-deficient (ob/ob) mouse: a new animal model of peripheral neuropathy of type 2 diabetes and obesity. Diabetes 55, 3335-3343.

Eckel, R. H., Grundy, S. M., and Zimmet, P. Z. (2010). The metabolic syndrome. Lancet 365, 1415-1428.

Fabbrini, E., Magkos, F., Mohammed, B. S., Pietka, T., Abumrad, N. A., Patterson, B. W., Okunade, A., and Klein, S. (2009). Intrahepatic fat, not visceral fat, is linked with metabolic complications of obesity. Proc. Natl. Acad. Sci. U.S.A. 106, 15430-15435.

Festi, D., Colecchia, A., Sacco, T., Bondi, M., Roda, E., and Marchesini, G.
(2004). Hepatic steatosis in obese patients: clinical aspects and prognostic significance. Obes. Rev. 5, 27-42.

Fielding, B. A., Callow, J., Owen, R. M. Samra, J. S., Matthews, D. R., and Frayn, K. N. (1996). Postprandial lipemia: the origin of an early peak studied by specific dietary fatty acid intake during sequential meals. Am. J. Clin. Nutr. 63, 36-41.

Folch, J., Lees, M., and Sloane Stanley, G. H. (1957). A simple method for the isolation and purification of total lipids from animal tissues. J. Biol. Chem. 497-508.

Friedman, J. M., and Halaas, J. L. (1998). Leptin and the regulation of body weight in mammals. Nature 395 , 763-770.

Haidari, M., Leung, N., Mahbub, F., Uffelman, K. D., Kohen-Avramoglu, R., Lewis, G. F., and Adeli, K. (2002). Fasting and postprandial overproduction of intestinally derived lipoproteins in an animal model of insulin resistance: evidence that chronic fructose feeding in the 
hamster is accompanied by enhanced intestinal de novo lipogenesis and ApoB48-containing lipoprotein overproduction. J. Biol. Chem. 277, 31646-31655.

Humason, G. L. (1972). Animal Tissue Techniques, 3rd Edn. San Francisco, CA: W.H. Freeman and Company.

Iqbal, J., Li, X., Chang, B., Chan, L., Schwartz, G. J., Chua, S. C., and Hussain, M. M. (2010). An intrinsic gut leptin-melanocortin pathway modulates intestinal microsomal triglyceride transfer protein and lipid absorption. J. Lipid Res. 51, 1929-1942.

Janardan, R. K., and Rao, M. S. (2006). Lipid metabolism and liver inflammation. II. Fatty liver disease and fatty acid oxidation. Am. J. Physiol. Gastrointest. Liver Physiol. 290, G852-G858.

Ji, H., and Friedman, M. I. (2008). Reduced hepatocyte fatty acid oxidation in outbred rats prescreened for susceptibility to diet-induced obesity. Int. J. Obes. (Lond.) 32, 1331-1334.

Johnston, J., Rao, G., and Lowe, P. (1967). The separation of the $\alpha$ glycerophosphate and monoglyceride pathways in the intestinal biosynthesis of triglycerides. Biochim. Biophys. Acta 137, 578-580.

Lagakos, W. S., Gajda, A. M., Agellon, L., Binas, B., Choi, V., Mandap, B., Russnak, T., Zhou, Y. X., and Storch, J. (2011). Different functions of intestinal and liver-type fatty acidbinding proteins in intestine and in whole body energy homeostasis. Am. J. Physiol. Gastrointest. Liver. Physiol. 300, G803-G814.

Lee, B., Zhu, J., Wolins, N. E., Cheng, J., and Buhman, K. K. (2009). Differential association of adipophilin and TIP47 proteins with cytoplasmic lipid droplets in mouse enterocytes during dietary fat absorption. Biochim. Biophys. Acta 1791, 1173-1180.

Lewis, G. F., O’Meara, N. M., Soltys, P. A., Blackman, J. D., Iverius, P. H.,
Druetzler, A. F., Getz, G. S., and Polonsky, K. S. (1990). Postprandial lipoprotein metabolism in normal and obese subjects: comparison after the vitamin A fat-loading test. J. Clin. Endocrinol. Metab. 71, 1041-1050.

Li, M., Paran, C., Wolins, N. E., and Horowitz, J. F. (2011). High muscle lipid content in obesity is not due to enhanced activation of key triglyceride esterification enzymes or to the suppression of lipolytic proteins. Am. J. Physiol. Endocrinol. Metab. 300, E699-E707.

Loirdighi, N., Ménard, D., and Levy, E. (1992). Insulin decreases chylomicron production in human fetal small intestine. Biochim. Biophys. Acta 1175, 100-106.

Mansbach, C. M., and Dowell, R. (2000). Effect of increasing lipid loads on the ability of the endoplasmic reticulum to transport lipid to the Golgi. J. Lipid. Res. 41, 605-612.

Mekki, N., Christofilis, M. A., Charbonnier, M., Atlan-Gepner, C., Defoort, C., Juhel, C., Borel, P., Portugal, H., Pauli, A. M., Vialettes, B., and Lairon, D. (1999). Influence of obesity and body fat distribution on postprandial lipemia and triglyceride-rich lipoproteins in adult women. J. Clin. Endocrinol. Metab. 84, 184-191.

Memon, R. A., Grunfeld, C., Moser, A. H., and Feingold, K. R. (1994). Fatty acid synthesis in obese insulin resistant diabetic mice. Horm. Metab. Res. 26, 85-87.

Nadler, S. T., Stoehr, J. P., Schueler, K. L., Tanimoto, G., Yandell, B. S., and Attie, A. D. (2000). The expression of adipogenic genes is decreased in obesity and diabetes mellitus. Proc. Natl. Acad. Sci. U.S.A. 97, 11371-11376.

Pan, D. A., Lillioja, S., Kriketos, A. D., Milner, M. R., Baur, L. A., Bogardus, C., Jenkins, A. B., and Storlien, L. H. (1997). Skeletal muscle triglyceride levels are inversely related to insulin action. Diabetes 46, 983-988.

Petro, A. E., Cotter, J., Cooper, D. A., Peters, J. C., Surwit, S. J., and
Surwit, R. S. (2004). Fat, carbohydrate, and calories in the development of diabetes and obesity in the C57BL/6J mouse. Metab. Clin. Exp. 53, 454-457.

Phan, C. T., and Tso, P. (2001). Intestinal lipid absorption and transport. Front. Biosci. 6, D299-D319.

Rasouli, N., Molavi, B., Elbein, S. C., and Kern, P. A. (2007). Ectopic fat accumulation and metabolic syndrome. Diabetes Obes. Metab. 9, 1-10.

Robertson, M. D., Parkes, M., Warren, B. F., Ferguson, D. J., Jackson, K. G. Jewell, D. P., and Frayn, K. N. (2003). Mobilisation of enterocyte fat stores by oral glucose in humans. Gut 52, 834-839.

Storch, J., Zhou, Y. X., and Lagakos, W. S. (2008). Metabolism of apical versus basolateral sn-2-monoacylglycerol and fatty acids in rodent small intestine. J. Lipid. Res. 49, 1762-1769.

Swift, L. L., Jovanovska, A., Kakkad, B. and Ong, D. E. (2005). Microsomal triglyceride transfer protein expression in mouse intestine. Histochem. Cell Biol. 123, 475-482.

Tobin, V., Le Gall, M., Fioramonti, X., Stolarczyk, E., Blazquez, A. G., Klein, C., Prigent, M., Serradas, P., Cuif, M. H., Magnan, C., Leturque, A., and Brot-Laroche, E. (2008). Insulin internalizes GLUT2 in the enterocytes of healthy but not insulin-resistant mice. Diabetes 57, 555-562.

Tso, P., Balint, J. A., and Rodgers, J. B. (1980). Effect of hydrophobic surfactant (Pluronic L-81) on lymphatic lipid transport in the rat. Am. J. Physiol. Gastrointest. Liver Physiol. 239, G348-G353.

Unger, R. H., Zhou, Y. T., and Orci, L. (1999). Regulation of fatty acid homeostasis in cells: novel role of leptin. Proc. Natl. Acad. Sci. U.S.A. 96, 2327-2332.

Windmueller, H. G., and Spaeth, A. E. (1978). Identification of ketone bodies and glutamine as the major respiratory fuels in vivo for postabsorptive rat small intestine. J. Biol. Chem. 253, 69-76.

Yamazaki, T., Sasaki, E., Kakinuma, C. Yano, T., Miura, S., and Ezaki, O. (2005). Increased very low density lipoprotein secretion and gonadal fat mass in mice overexpressing liver DGAT1. J. Biol. Chem. 280, 21506-21514.

Zhang, Y., Proenca, R., Maffei, M., Barone, M., Leopold, L., and Friedman, J. M. (1994). Positional cloning of the mouse obese gene and its human homologue. Nature 372, 425-432.

Zhu, J., Lee, B., Buhman, K. K., and Cheng, J. (2009). A dynamic, cytoplasmic triacylglycerol pool in enterocytes revealed by ex vivo and in vivo coherent anti-Stokes Raman scattering imagings. J. Lipid Res. 50, 1080-1089.

Conflict of Interest Statement: The authors declare that the research was conducted in the absence of any commercial or financial relationships that could be construed as a potential conflict of interest.

Received: 10 October 2011; accepted: 03 February 2012; published online: 24 February 2012.

Citation: Douglass JD, Malik N, Chon S-H, Wells K, Zhou YX, Choi AS, Joseph $L B$ and Storch $J$ (2012) Intestinal mucosal triacylglycerol accumulation secondary to decreased lipid secretion in obese and high fat fed mice. Front. Physio. 3:25. doi: 10.3389/fphys.2012.00025

This article was submitted to Frontiers in Fatty Acid and Lipid Physiology, a specialty of Frontiers in Physiology. Copyright (c) 2012 Douglass, Malik, Chon, Wells, Zhou, Choi, Joseph and Storch. This is an open-access article distributed under the terms of the Creative Commons Attribution Non Commercial License, which permits noncommercial use, distribution, and reproduction in other forums, provided the original authors and source are credited. 